\title{
ACTIVATION AND DEACTIVATION PROCESSES IN THE POSTPONED INTENTION PARADIGM
}

\author{
Juan J. G. MEILÁN \\ University of Salamanca, Spain
}

\begin{abstract}
This study analyzes the intention superiority effect (ISE). The ISE (Goschke \& Kuhl, 1993) is defined as faster access time to the contents related to information learned as a part of a to-be-executed task (prospective memory) than to information learned not as a part of a to-be executed task (neutral contents). The experiment is a replica of the ISE using the Goschke and Kuhl procedure. The ISE was replicated in the measure prior to the execution of the action. Furthermore, the ISE disappeared after the execution of the intention.
\end{abstract}

Key words: motivation, intention, prospective memory, intentional superiority effect

The intention superiority effect (ISE) is described as the persisting activation that the representation of an intention has in declarative memory in the retention period of an intention (Goschke \& Kuhl, 1993). According to this proposal, the representation of an intentional activity is characterized by a higher or more sustained level of activation in the memory as compared to the representation of a similar non-intentional activity.

To analyse the ISE, Goschke \& Kuhl (1993) designed the delayed intention paradigm. The experimental condition was one in which the participants learned pairs of scripts representative of the two common tasks in everyday life (i.e. clear a desk). The participants were then instructed to later perform one of the scripts (intentional script). The other script becomes neutral. Prior to the retrieval and execution of intentional script, the explicit access to the contents of both scripts was measured. Goschke \& Kuhl (1993) found that the latencies in recognising the words related to a later to-be-executed action script (prospective script) were faster compared to words from a not to-be-executed action script (neutral script). The results were interpreted in relation to the intrinsic property of intentions to decline more slowly in the declarative memory than the representation of non-intentional activity.

The study by Goschke and Kuhl has had a great impact in recent years and has been extensively cited (see review in Brandimonte, Einstein \& McDaniel, 1996). Different studies have appeared which have addressed the phenomenon (i.e., Dockree and Ellis, 2001; Freeman \& Ellis, 2003; Marsh, Hicks, \& Bink, 1998; Maylor, Darby, \& Della Sala, 2000).

In this study, I used the delayed intention paradigm in order to explore the generality

Correspondence concerning this article should be addressed to J. J. G. Meilán, Department of Basic Psychology, University of Salamanca, Avda. de la Merced, 109-131, 37005 Salamanca. Spain (e-mail: meilan@usal.es). 
of the ISE. The experiment was a replication of the original studies by Goschke \& Kuhl (1993). However, some modifications have been introduced in order to investigate some of its specific properties. The modifications were introduced concerning the type of material and procedure used.

First, an event-based prospective memory task was used as the intention modality (Einstein \& McDaniel, 1990). This task was used in order to be more consistent with the literature, in which more studies have used event-based rather than activity-based tasks. The participants expected to have to remember to perform the task when a certain event occurred (i.e. when a tone sounded). This normally requires the interruption of the ongoing activity. In the Goschke and Kuhl study, the participants expected to execute the task when they finished the recognition test. It is an activity-based prospective memory task.

Second, we replicated the study of Goschke and Kuhl using material less sensitive to semantic category effects. In the Goschke \& Kuhl study, the scripts consisted of highly semantically-related actions, i.e., the actions were represented by a set of words belonging to the same semantic category. The intention could activate the category related with the title. In my study, the intentions were represented as scripts in the formal sense introduced by Schank \& Abelson (1977): each sentence of the scripts represented a typical action. Each word in the sentence (especially the verbal forms that indicated actions of a general type, such as "look for", etc.) was not necessarily semantically associated with the script title. That is, each word was learned in the context of a certain action.

In addition to questions concerning the procedure, an attempt was made to address other questions related to the activation of the intentional representation. According to Goschke \& Kuhl (1993), the ISE is consistent with Anderson's ACT* model (1983). In the ACT* model, the goals for an immediate action in working memory are represented as source nodes that maintain their activation level without rehearsal. Goschke \& Kuhl (1993) expanded this idea by conceiving intentions as subthreshold source nodes, but in long-term memory. However, Marsh, Hicks \& Bink (1998) proposed that the intentions provide an internal context marker. This marker is triggered once its retrieval condition has been satisfied. In this study, an attempt was made to investigate the mechanisms underlying the activation of intentions.

In the experiment, the recognition test was administered before and after participants had carried out (executed) the instruction. The goal of the second recognition test was to verify what happens with the ISE once the corresponding intention has been executed (i.e. physically carried out). If the intention reflects activation, the representation of intentions might be expected to lose its privileged status in the memory after the participants had executed the task.

The goal of study was to investigate whether the representation of a to-be-executed script would persist stored at stronger and longer-lasting levels of activation in memory than the comparable representation of a neutral script.

It was expected that, in the execution condition, the prospective words would be recognised reliably faster compared with neutral words. Therefore, and from the basic supposition of an inverse relationship between latency and activation (e.g., Anderson, 
1983; Ratcliff \& McKoon, 1978), the results could be interpreted as a greater activation of the intentional content stored in long term memory versus neutral contents that do not directly affect the person. These differences were not expected in the observation condition. In this condition, the prospective script has to be executed by the experimenter.

The recognition latencies were measured twice: before and after the subject executed the prospective task. The goal of the second measurement was to verify what happens with the supposedly greater activation of the intentional content once the corresponding intention has been carried out. If quicker access to the intentional words is due to the ISE, this effect should disappear once the task has been completed. In the second measurement, it was expected that the recognition latencies of the words from the to-beexecuted script should not be different from the recognition latencies of the words from the not-to-be-executed script.

This hypothesis would contradict the results obtained in the studies on the retrospective recall of already performed actions (see review in Jahn \& Engelkamp, 2003). In these studies it was found that actions involving motor activity are better recognised than other types of actions, which have been learned verbally but not executed. Thus, the hypothesis in agreement with these studies is that recognition latencies will continue to be shorter for words related to the intention after it has been enacted, but now as a consequence of its motor execution.

\section{METHOD}

\section{Participants}

Thirty-two students participated voluntarily in the experiment. They were chosen at random from second year Psychology students at the University of Salamanca ( 29 women and 3 men; aged between 18 and 22; mean age 19.34 years). The participants carried out two experimental sessions. Two of the participants were eliminated from the analyses given because their scores, in the second experimental session, exceeded the mean scores of the other participants by 2.5 standard deviations, which led us to think that their collaboration in the second session was not appropriate. Thus, the final sample was made up of 30 participants.

\section{Material}

The experimental scripts were grouped in two pairs, the A and B versions of each script. The presentation of each pair was counter-balanced. Each of the experimental scripts (see appendix 1 for the English translation of the Spanish scripts) was made up of the title ("Go to see the doctor" and "Go to a restaurant") and six sentences referring to simple actions. The sentences in Spanish were of the type: verb (action) + noun. These four short scripts were prepared based on the complete scripts that were obtained in a previous normative study (Meilán \& Mateos, 2002). We choose the actions that received high scores for typicalness in relation to the script.

The experimental list of words (48 words) for each participant was made up of the 24 words from the two scripts they had studied (i.e., the A version of each script) and the 24 words from the other two, which acted as distracters (i.e., the B version of each script). Each word in the sentence was not necessarily semantically associated with the script title. In a preview normative study, a group of 17 participants were asked to evaluate the semantic relation between the words and the title script. The mean score of the semantic relation was 4, 17 for verbs and 5, 66 for nouns on a scale of 1 to 7 (see appendix 1, semantic relation values for each script).

The participants could execute the actions of each script in the corresponding simulated scenarios 
provided with the necessary objects, including the characters in the script with which the participant had to interact. The characters are articulate puppets, which doctors, nurses, waiters, etc. represent.

\section{Design and Procedure}

The experimental procedure was composed of two parallel experimental sessions. Each session consisted of four phases.

First, in the study phase, the participants had to learn a pair of the two simple everyday tasks according to a very strict learning criterion. Both script titles ("Go to see the doctor" and "Go to a restaurant"), were presented alternately at least three times. In each presentation the actions of each script appeared on the computer screen one by one consecutively (each action for 6 seconds). When the sequence ended, the complete script appeared made up of the title and the 6 actions (for 30 seconds). Next, the participant carried out a distraction task for 45 seconds.

Second, the participant received a screen notifying the later appearance of experimental instruction. This screen indicated to the participants that they could not carry out the instruction immediately, but would have to do so later when they heard a warning tone on the computer. Until the warning tone appeared the participants were asked to perform a memory test, which consisted of recognizing the words from the two scripts studied. After this screen, they received the experimental instruction. Depending on the experimental condition, one of the two instructions appeared on the monitor for 3 seconds. In the experimental execution condition, the participants were informed that they would have to execute one of the scripts later, whereas in the observation condition, they were instructed to observe the experimenter performing one of the scripts later and note the mistakes. Depending on the experimental condition, the participants received the instruction "Execute" or "Observe", followed by the title of the prospective script. Immediately under this appeared the message "Do not execute" or "Do not observe", followed by the title of the neutral script. Half of the participants were randomly assigned to the execution condition, and the other half to the observation condition.

Third, immediately after the instruction the participants were given a word recognition test. After ten buffer words, the experimental items began (24 experimental and 24 distracter words). The 48 words appeared in a random order per participant. The order was randomised by the computer. Each word on the test list was presented on the CRT screen one at a time and remained there until the participant gave a response. After correct responses, a blank interval appeared for $200 \mathrm{~ms}$ followed by a fixation point for 500 $\mathrm{ms}$ and the presentation of the next word. After incorrect responses, the word error was presented for 2 seconds to prevent the negative effects that the mistake could have on the following word. After that the blank interval and fixation point appeared. The recognition test was programmed in such a way that 48 experimental words appeared. At the end of the test list, an audible warning tone sounded on the computer for 3 seconds. After that, another five buffer words appeared.

Finally, upon hearing the warning tone, the participants had to indicate to the experimenter that they needed to execute the script (execution condition) or they needed to observe how accurate the experimenter was at executing the script (observation condition). The participants then moved on to another part of the laboratory to execute or observe the experimenter executing the prospective script. If the participant continued to answer after the signal (4 people) or did not correctly remember the instruction ( 2 people: one had forgotten which script was the prospective one and one had not seen it), they were eliminated from the analyses and they were replaced by their substitutes (taken from the original randomised list).

The second experimental session, carried out after an interval of 30 days, was parallel to the first. In the second experimental session, the participants received the experimental instruction opposite to the one that they had received in the first session (execute versus observe). The two scripts studied were the same as in the previous session and each student was assigned, as a prospective script, the same one that he/she had had by chance in the first session. Furthermore, once the participants finished the prospective task in the second session, they were asked to do the recognition test again. Therefore, the recognition latencies for the words were obtained after the intention was performed. 
Table 1. Mean and Standard Deviations of the Latencies of Recognition of the Studied Words According to the Instruction and the Word Type in Experiment 1.

\begin{tabular}{|c|c|c|c|c|c|c|c|c|}
\hline \multirow{4}{*}{ Instruction } & \multicolumn{4}{|c|}{ Pre-intentional measure } & \multicolumn{4}{|c|}{ Post-intentional measure } \\
\hline & \multicolumn{4}{|c|}{ Type of word } & \multicolumn{4}{|c|}{ Type of word } \\
\hline & \multicolumn{2}{|c|}{ Prospective } & \multicolumn{2}{|c|}{ Neutral } & \multicolumn{2}{|c|}{ Prospective } & \multicolumn{2}{|c|}{ Neutral } \\
\hline & R.T. & $\mathrm{A}^{\prime}$ & R.T. & $\mathrm{A}^{\prime}$ & R.T. & $\mathrm{A}^{\prime}$ & R.T. & $\mathrm{A}^{\prime}$ \\
\hline Execution & 890 & 0.78 & 964 & 0.75 & 863 & 0.80 & 894 & 0.80 \\
\hline Observation & 940 & 0.83 & 925 & 0.78 & 807 & 0.90 & 820 & 0.90 \\
\hline
\end{tabular}

\section{RESULTS}

\section{Results of the Recognition Test During the Retention Interval}

The experimental variables were instruction (execution vs. observation) and item type (prospective vs. neutral), both of which were measured within-subjects. The dependent variable was recognition latencies. The trials reflecting incorrect responses $(4.36 \%)$ or latencies beyond 2.5 SDs of a given participant's mean $(0.65 \%)$ were excluded from the statistical analysis. The means of the remaining response times for correct responses were only computed for each participant in each experimental condition. These means were the basis for the following analyses: A 2 (instruction) $\times 2$ (type of item) ANOVA within-subject factorial design was used.

This analysis showed the interaction effect between the two independent variables, $F(1,29)=8.05, M S E=59853.323, p<0.01$. No principal effects were detected that were due to either of the two independent variables, instruction, $F(1,29)=0.02$, $M S E=874.800$, or item type, $F(1,29)=3.08, M S E=25114.133$. Simple comparison analysis showed that, in the execution condition, the prospective words were recognised reliably faster $(890 \mathrm{~ms})$ as compared with neutral words $(964 \mathrm{~ms}), F(1,58)=10.42$, $M S E=81254.400, p<0.01,(\mathrm{dfadj} .=57.87)$. No differences were found between prospective and neutral words in the observation condition, $F(1,58)=0.48$, $M S E=3713.067$. However, the comparisons between the execution and observation conditions did not reveal significant differences between the prospective words, $F(1$, $58)=1,73, M S E=37600.067$ or the neutral ones, $F(1,58)=1.06, M S E=23128.067$ (dfadj. $=40.48)$. Table 1 shows the mean response times for words from the prospective and neutral scripts in the execution and observation conditions.

It was necessary to rule out the possibility of the shorter latency times for the prospective words in the execution condition being due to a lower performance of the subject in these words (a mere exchange between speed and accuracy). Because of the limited number of observations per subject (the number of errors was very low: mean of 4 errors per participant), a nonparametric equivalent to d', the discriminability index A' was used (see Donaldson, 1992). The same $2 \times 2$ ANOVA was used with $\mathrm{A}^{\prime}$ as the dependent 
variable. No interaction effect was found between the instruction variable and the type of item variable, $F(1,31)=0.03, M S E=0.000153$. No principal effects were found to be due to the instruction variable, $F(1,31)=1.03, M S E=0.018528$ or to the type of item variable, $F(1,31)=2.73, M S E=0.019503$. Therefore, we can conclude that the greater speed of access to the words related to an intention did not entail a reduction in the discriminability of these words (see table 1).

These results replicated Goschke \& Kuhl's (1993) findings. The participants recognised the prospective words from the to-be-executed script faster than the neutral words. The prospective words from the to-be-executed script seem to be more activated in long-term memory than other kinds of words from the not-to-be-executed neutral script. This greater activation is not due to the activation of the semantic category related to the task, but to the activation of individual words that formed part of the representation of an intended action. Marsh, Hicks \& Bryan (1999) found similar ISE with tasks made up of a constellation of unrelated activities. However, in my study, some of the words clearly appeared to be semantically related to the script header. As we have seen in appendix 1, the nouns were more semantically related to the script title than were the verbs. To investigate the influence of the modality of the words (nouns and verbs) on the obtained results, we carried out an ANOVA $2 \times 2 \times 2$ in which the variable "word modality" was introduced. The ANOVA proved that no interaction effect existed among the three variables (Instruction, Item Type and Word Modality), $F(1,29)=0.05, M S E=493.067$. Nor were there interaction effects between Instruction and Word Modality, $F(1$, $29)=0.11, M S E=2136.067$, or between Item Type and Word Modality, $F(1,29)=0.68$, $M S E=15232.267$. The interaction effect only persisted between Instruction and Item Type, $F(1,29)=5.64, M S E=93852.150, p<0.05$. Therefore, the differences in the semantic relationship of the words, whether they were nouns or verbs, did not affect the results obtained.

\section{Results of the Recognition Test Once the Intention Had Been Carried Out}

In the second session, the participants were asked to do the recognition test again once they had finished the execution of instruction (the execute task or observe task). A $2 \times 2$ ANOVA was carried out with the factors "type of script" (prospective versus neutral) of the within-subject type, and "preview instruction" (execution vs. observation) of the between-subjects type. No interaction effect was found between the two variables $F(1,30)=0.28, M S E=1406.250$. Neither the main effect of instruction $F(1,30)=1.48$, $M S E=67340.250$ nor the type of item $F(1,30)=1.51, M S E=7656.250$ were significant. After the script was executed, the participants did not recognise words from the to-beexecuted prospective script faster than words from the neutral script. Again, the index A' was used to calculate the differences in word discriminability (see, table 1). No interaction effect was found between the two variables $F(1,30)=0.45, M S E=0.0012$. Neither the main effect of instruction $F(1,30)=0.35, M S E=0.0045$ nor the item type $F(1,30)=1.80, M S E=0.0049$ were significant.

These results were similar to previous research on the ISE using a lexical-decision task (Marsh, Hicks \& Bink, 1998). They found that once the task had been done, the 
representation of prospective script was reliably slower than for the representation of neutral script. They interpreted that, after performing and intention, the representation of the intention rapidly loses activation; the representation decays in the memory or is actively inhibited. In our study, using an explicit recognition test, this inhibitory phenomenon was not found (neither did we find this inhibitory effect in Meilán \& Carro, 2004). The results were thus favourable to the hypothesis of a deactivation of the intentional representation once the intention had been carried out.

\section{DISCUSSION}

The importance of the so-called intention superiority effect (Goschke \& Kuhl, 1993) has been corroborated in the study using typical actions within a script and a recognition memory test. The study replicated the finding of an ISE before the execution of the prospective task and, in addition, showed that the ISE disappears after the execution of the intention. Thus, the activation of a to-be-executed script is not semantic-category activation, but rather an activation of words learned in the context of a certain action. From a theoretical point of view, the pattern of results obtained fits the ACT* model (Anderson, 1983; Lebiere \& Lee, 2002) and was entirely consistent with the Goschke \& Kuhl interpretation (1996; and other authors such as Mäntylä, 1996). There is a greater activation of the intentional content stored in long-term-memory in comparison to neutral information. The intentions were represented as goal nodes, which conferred them additional activation.

Therefore, the overall level of activation for items in the memory at any given time is fairly constant. When the prospective script is in a heightened state of activation in the memory, the neutral script is in a diminished state of activation in the memory. However, when the prospective script is executed (the intention is completed), the activation of items in the memory is distributed unevenly between the neutral and prospective memory.

\section{REFERENCES}

Anderson, J. R. 1983. The architecture of cognition. Cambridge, MA: Harvard University Press.

Brandimonte, M., Einstein, G. O., \& McDaniel, M. A. (Eds.). 1996. Prospective memory: Theory and applications. Mahwah: Erlbaum

Dockree, P. M., \& Ellis, J. A. 2001. Forming and cancelling everyday intentions: Implications for prospective remembering. Memory \& Cognition, 29, 1139-1143.

Einstein, G. O., \& McDaniel, M. A. 1990. Normal aging and prospective memory. Journal of Experimental Psychology: Learning, Memory, \& Cognition, 16, 717-726.

Freeman, J. E., \& Ellis, J. A. 2003. The intention-superiority-effect for naturally occurring activities: the role of intention accessibility in everyday prospective remembering in young and older adults. International Journal of Psychology, 38, 215-228.

Goschke, T., \& Kuhl, J. 1993. Representation of intention: Persisting activation in memory. Journal of Experimental Psychology: Learning, Memory \& Cognition, 19, 1211-1226

Goschke, T., \& Kuhl, J. 1996. Remembering what to do: Explicit and implicit memory for intentions. In M. Brandimonte, G. O. Einstein \& M. A. McDaniel (Eds.), Prospective memory: Theory and applications 
(pp. 53-91). Mahwah: Lawrence Erlbaum.

Jahn, P., \& Engelkamp, J. 2003. Design-effects in prospective and retrospective memory for actions. Experimental Psychology, 50, 4-15.

Lebiere, Ch., \& Lee, F. J. 2002. Intention superiority effect: A context-switching account. Cognitive Systems Research, 3, 57-65.

Mäntylä, T. 1996. Activating actions and interrupting intentions: Mechanisms of retrieval sensitization in prospective memory. In M. Brandimonte, G. O. Einstein \& M. A. McDaniel (Eds.), Prospective memory: Theory and applications (pp. 93-113). Mahwah, NJ: Erlbaum.

Marsh, R. L., Hicks, J. L., \& Bink, M. L. (1998). Activation of completed, uncompleted, and partially completed intentions. Journal of Experimental Psychology: Learning, Memory, \& Cognition, 24, 350361.

Marsh, R. L., Hicks, J. L., \& Bryan, E. 1999. The activation of unrelated and cancelled intentions. Memory \& Cognition, 27, 320-327.

Maylor, E. A., Darby, R., \& Della Salla, S. 2000. Retrieval of performed versus to-be-performed tasks: A naturalistic study of the intention-superiority effect in normal aging and dementia. Applied Cognitive Psychology, 14, S83-S98.

Meilán, J. J. G., \& Carro, J. 2004. Shopping as an example of intention memory: the sequence in the execution of a prospective task and its predictors. Psicología General y Aplicada, 57, 343-360.

Meilán, J. J. G., \& Mateos, P. M. 2002. Schemes in Spanish: the representation, in the form of scripts, of five daily tasks by young people and elderly people. Revista de Psicología General y Aplicada, 55, 161185.

Ratcliff, R., \& McKoon, G. 1978. Priming in item recognition. Evidence for the propositional structure of sentences. Journal of Verbal Learning \& Verbal Behaviour, 17, 403-417.

Schank, R. C., \& Abelson, R. 1977. Scripts, plans, goals and understanding. Hove, UK: Erlbaum.

(Manuscript received May 16, 2006; Revision accepted August 28, 2007) 
APPENDIX 1. The English translation of the Spanish scripts.

\begin{tabular}{|c|c|c|c|}
\hline SCRIPT & ACTIONS & Typical & VALUES \\
\hline GO TO DOCTOR & To ask the time & 5.29 & Actions typicality: 5.411 \\
\hline \multirow{5}{*}{ VERSION A: } & To follow the nurse & 5.51 & Verbs semantic relation: 2.62 \\
\hline & To enter the surgery & 5.77 & Nouns semantic relation: 5.33 \\
\hline & To be examined & 5.13 & Words: 19 \\
\hline & To receive a diagnosis & 5.55 & Syllables: 33 \\
\hline & To clarify doubts & 5.21 & Latencies D.L. Mean $619.33 \mathrm{~ms}$ \\
\hline GO TO DOCTOR & To wait your turn & 5.60 & Actions typicality: 5.531 \\
\hline \multirow{5}{*}{ VERSION B } & To greet the doctor & 5.79 & Verbs semantic relation: 3.52 \\
\hline & To tell your symptoms & 5.47 & Nouns semantic relation: 5.67 \\
\hline & To lie down on the stretcher & 4.92 & Words: 18 \\
\hline & To show your national health card & 5.85 & Syllables: 34 \\
\hline & To collect the prescription & 5.55 & Latencies D.L. Mean $631 \mathrm{~ms}$ \\
\hline \multirow{2}{*}{$\begin{array}{l}\text { GO TO } \\
\text { RESTAURANT }\end{array}$} & to look for a seat & 5.50 & Actions typicality: 5.433 \\
\hline & to order the set menu & 5.38 & Verbs semantic relation: 3.92 \\
\hline \multirow[t]{4}{*}{ VERSION A: } & to be served a drink & 5.19 & Nouns semantic relation: 6.00 \\
\hline & to begin lunch & 5.61 & Words: 18 \\
\hline & to converse after dinner & 5.41 & Syllables: 34 \\
\hline & to put on your coat & 5.49 & Latencies D.L. Mean $611.92 \mathrm{~ms}$ \\
\hline \multirow{2}{*}{$\begin{array}{l}\text { GO TO } \\
\text { RESTAURANT }\end{array}$} & to choose the table & 5.39 & Actions typicality: 5.367 \\
\hline & to call the waiter & 5.15 & Verbs semantic relation: 4.82 \\
\hline \multirow[t]{4}{*}{ VERSION B } & to order the food & 5.46 & Nouns semantic relation: 6.50 \\
\hline & to eat dessert & 5.51 & Words: 17 \\
\hline & to pay the bill & 5.94 & Syllables: 31 \\
\hline & to leave a tip & 4.94 & Latencies D.L. Mean $601 \mathrm{~ms}$ \\
\hline
\end{tabular}

